\title{
Design and control of three fingers motion for dexterous assembly of compliant elements
}

\author{
W. Widhiada ${ }^{1^{*}, 2}$, S.S. Douglas ${ }^{1}$, I.D.Jenkinson ${ }^{1}$ and J.B. Gomm ${ }^{1}$ \\ ${ }^{1}$ School of Engineering Liverpool John Moores University, Byrom Street,Liverpool, L3 3AF, UK \\ ${ }^{2}$ Department of Mechanical Engineering, Udayana University, Denpasar, Bali, INDONESIA \\ "Corresponding Author: e-mail: W.Widhiada@2008.ljmu.ac.uk, Tel +44-1512312125
}

\begin{abstract}
In this paper, the authors describe and demonstrate how a three fingered gripper can be designed and simulated to provide both gross motion and fine motion to the fingers. Satisfactory motion responses for the finger simulation are achieved. The fine motion including force feedback and the gross motions, which orientate the fingers into their approximate configuration are provided by a classical PD control strategy. The force controlling gripper in contact with the environment is very important in industry applications. The fingers are to be controlled in a manner which mimics the kinematics and dynamics of the thumb force finger and index finger of a human hand. This mimicry is required to design the correct motions and tactile forces necessary to handle delicate and non delicate engineering components. In order to evaluate the design philosophy and capability of the three fingered gripper, a challenging assembly process has been identified. This is assembly of a gas regulator valve which is currently being manually assembled since the dexterous motions of the human hand out perform current automatic assembly strategies. The three fingered gripper assembly was built using Solidworks software tool, and it's mechanical assembly representation was established in SimMechanics.
\end{abstract}

Keywords: Three Fingers Gripper, PD control, Gross Motion, Fine Motion, Tactile Force, Mimicry.

DOI: http://dx.doi.org/10.4314/ijest.v3i6.2S

\section{Introduction}

Robotic hands have been developed with the aim of copying the human hand in terms of dexterity and adaptive capabilities to function as either a manipulator or as a prosthetic device. Pioneer designs include: Okada's hand (Okada, 1982) the Stanford/JPL hand (Salisbury et al, 1982), the Belgrade/USC hand (Bekey et al, 1999), the Utah/MIT Dexterous hand (Jacobsen, 2001) and the LMS hand (Gazeau, 2001). However, significant efforts have still to be made to find designs simple enough to be easily built and controlled, in order to obtain practical systems (Bicchi, 2000). To overcome the limited success of the early hand designs, considerable emphasis has been placed in reducing the number of degrees of freedom (DOFs) without sacrificing dexterity and thereby reducing the required number of actuators. In particular, the SSL hand (Akin, 2002), the Graspar hand (Crisman et al, 1996), the DIES-DIEM hand (Biagotti et al, 2001), and the TBM hand (Dechev et al, 2001) have followed this path. There are a few prototypes which use a smaller number of actuators but do not decrease the number of DOFs. This approach, known as under actuation can be implemented through the use of passive elements including springs to a mechanical adaptation of the finger to the shape of the object to be grasped (Hirose et al, 1978) - ( Massa et al, 2002). A similar approach consists of using elastic phalanges, which increase the adaptation capability but decrease considerably the strength of the grasp (Schulz et al, 2001).

In a complex handling system, the gripper although small is a significant part of the entire system (Guerry, 2001). Grippers can be the most design-intensive components of an assembly system. Proper gripper design can simplify the overall assembly, increase the overall system reliability, as well as decrease the implementation cost. Whilst grippers have been widely used for automated manufacturing, assembly, and packing, etc, very little attention has been given to the research and design of grippers for the high speed assembly of compliant elements. Initial work in gripper design included a multitude of different gripper designs, including 
grippers with stiff fingers and/or flexible fingers, vacuum grippers, and magnetic grippers (Lunstrom, 1977). A mechanical hand has been developed based on the basic prehensile patterns of a human hand and the designed hand can grasp all basic shapes such as rectangular and triangular prisms, spheres and cylinders (Skinner, 1975). In Japan, significant work has been undertaken in which the shape, structure, and motion of the human hand is simulated for the design of grippers (Okada et al, 1977). The soft gripper has been developed that is able to conform the outer contour of objects of irregular shapes and hold them with uniform pressure distribution along the length of the fingers (Hirose et al, 1977). A creative approach to designing industrial grippers by using kinematic structure database, which contains general information about robot gripper mechanism, kinematic structures, function, type of drive mechanism and applications is presented (Lee, 1982). Classification system for grippers has been developed based on factors such as size, position and orientation of gripping forces. This method is used to determine additional design constraints based on the final installation and activities that the robot would be performing (Kolpanshnikov et al, 1979). The new anthropomorphic robot hand was designed as a dexterous end-effector that can cope with a wide variety of tasks and object encountered in its environment (Kyriakopoles et al, 1977).

Robot fingers and hands are usually confronted with a situation in which a few contact points of each finger take place simultaneously and must be maintained so that the captured object does not slip from the grasp. To this end, a number of methodologies to achieve stable control and dexterous manipulation tasks have been proposed, and a number of related studies published in the late 1980s. They also propose a geometric approach to determine the strength of this. Their study noted that the concepts "forced the closure of" (Reuleaux et al, 1983) and "form closure" (Lakshiminarayana, 1978) are essential for complete refrain from captured objects. Then, forcing the closure of system is formulated (Nguyen, 1986, 1987and1988).Therefore the strategy for computing optimal internal forces that can be explored actively. A direct-calculation method to capture the style and provide the optimal internal strength so that the internal dependency friction force on the fixed contact is minimized (Ji et al, 1988). The grasp forces required for stable grasping can be reduced to a linear programming problem that considers friction and joint torque limit constraints (Kerr et al, 1986). These studies, however, were based not on dynamics but on statics with kinematic and geometric description. An analytic grasp models, quality measures and constraints are used to solve the problems of choosing a grasp (Cutkosky, 1989 ). Furthermore, the kinematics of rolling between the object and fingertip surfaces using velocity and the normal constraints and systematically derived the dynamics of grasp containing the rolling task (Cole et al, 1989 ). In addition the optimal grasp of an object using differential geometry so that a task could be applied efficiently and robot motion planning could be found under nonholonomic rolling constraints (Li et al, 1988) and (Sastry et al, 1989). For the first time emphatically described grasping and manipulating the forces that can be used independently to control input applied to the robot hand (Kobayashi, 1985). Moreover, the architecture of control based on the decomposition schemes of grasping and manipulating forces in the presence of coulomb friction between the target object and the robot fingers (Yoshikawa et al, 1988) and (Nakamura et al, 1989 ) This fundamental type control scheme, such as independent grasping and manipulating motions or forces, has been clarified and is often employed by the robotics and automation researchers. Therefore, an extraordinary number of theoretical analyses of manipulators and robotic hands have been proposed, and their successful application in control strategy became a well known way to move manipulators and robotic hands. After the 1990s, these manipulation problems were further developed, and these control techniques were applied to a number of robots. Examples are: the learning force control for a position controlled robotic manipulator was established to compensate the uncertainties regarding stiffness of the constraint environment in an iterative manner (Qiao et al, 1999); the problems of force control by considering the force sensing method without changing the control architecture is solved and improved Seiichiro, (2007); a method for automatic grasp generation based on object shape primitives by demonstration (Johan, 2008); the simulation of a robotic manipulator in MATLAB/Simulink and visualization of robot systems using general dynamic engines and graphical languages (Leon, 2008); simulation of a multifinger robotic gripper of dexterous grasping (Ohol, 2008).

In this paper the authors describe how a three fingered gripper can be designed and simulated to provide both gross motion and fine motion to the fingers. The fine motion including force feedback and the gross motions which orientate the fingers into their approximate configuration is provided by a classical PD control strategy. The fingers are to be controlled in a manner which mimics the kinematics and dynamics of the thumb force finger and index finger of a human hand. This mimicry is required to design the correct motions and tactile forces necessary to handle delicate and non delicate engineering components. In order to evaluate the design philosophy and capability of the three fingered gripper challenging assembly process has been identified. This is the assembly of a gas regulator valve which is currently being assembled since the dexterous motions of the human hand out perform current automatic assembly strategies.

\section{Robot Finger Design}

In order to demonstrate the expediency of the described robot hand approach, we have developed a three fingered hand prototype by developing two fingers: index and middle finger and thumb in a 3 dimension model with Solidworks software (Widhiada et al, 2010). Actuators, position sensors and force sensors have been integrated in the hand structure.

2.1. Architecture of Robot Finger: The dexterous robot gripper is equipped with two identical fingers (index and middle finger) and a thumb. An actuator is installed in each joint of the fingers and thumb. The joints of the finger, the distal interphalangeal (DIP), proximal interphalangeal (PIP) and metacarpophalangeal (MCP) joints each have 1 DOF to provide a rotation movement. 
The prismatic sliding joints are also installed at each fingertip of the fingers and thumb. The architecture of the three fingers is show in Figure 1.

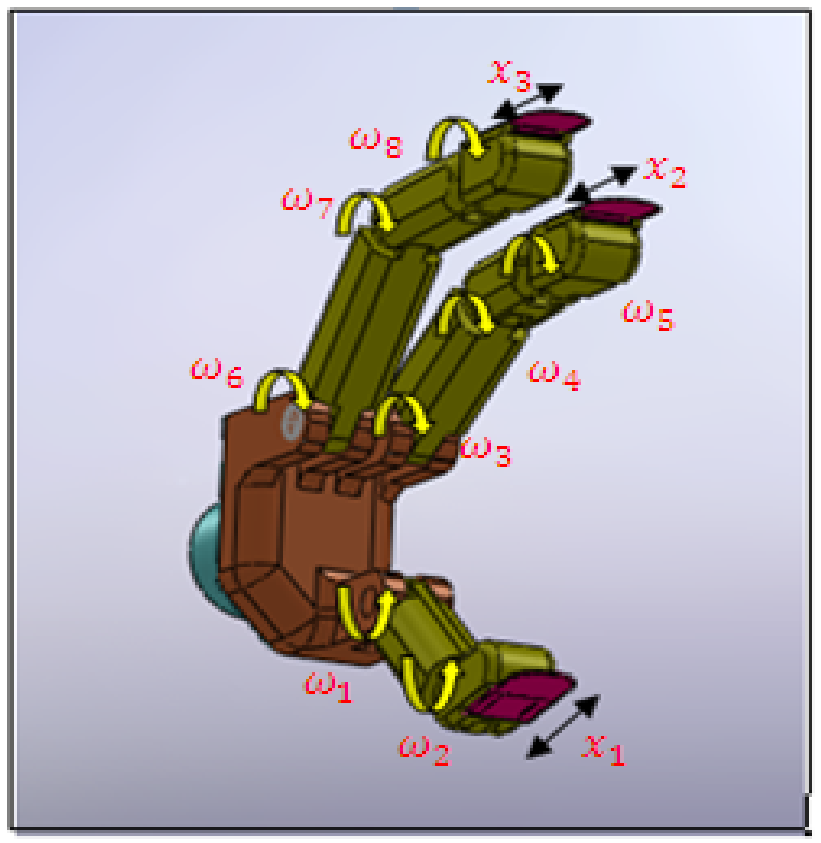

Figure 1. Architecture of Robot Gripper.

The thumb has been designed in order to complete the hand and it has three degrees of freedom. A prismatic sliding element is installed at the end of each finger to facilitate the handling of awkward small compliant geometries. The thumb is both able to flex and extend. The index and middle finger prototypes have been designed such that each finger has three degrees of freedom. The prismatic sliding joint is again installed at the end of the fingers to handle small and awkward objects. In order to simulate the finger motions each revolute joint has an associated Servo DC motor attached to it. The extension finger tip is also controlled using a DC motor Servo driving a rack and pinion.

2.2 Kinematic and Dynamic: Based upon considering the motion of the human finger a simplified robotic finger has been designed. This finger is equipped with four degrees of freedom, three revolute joints and the single prismatic sliding element at the end of the finger. Each body of the finger has masses attached that represent the revolute joints of the finger. The kinematics of the physical model of the thumb is shown in Figure 2.

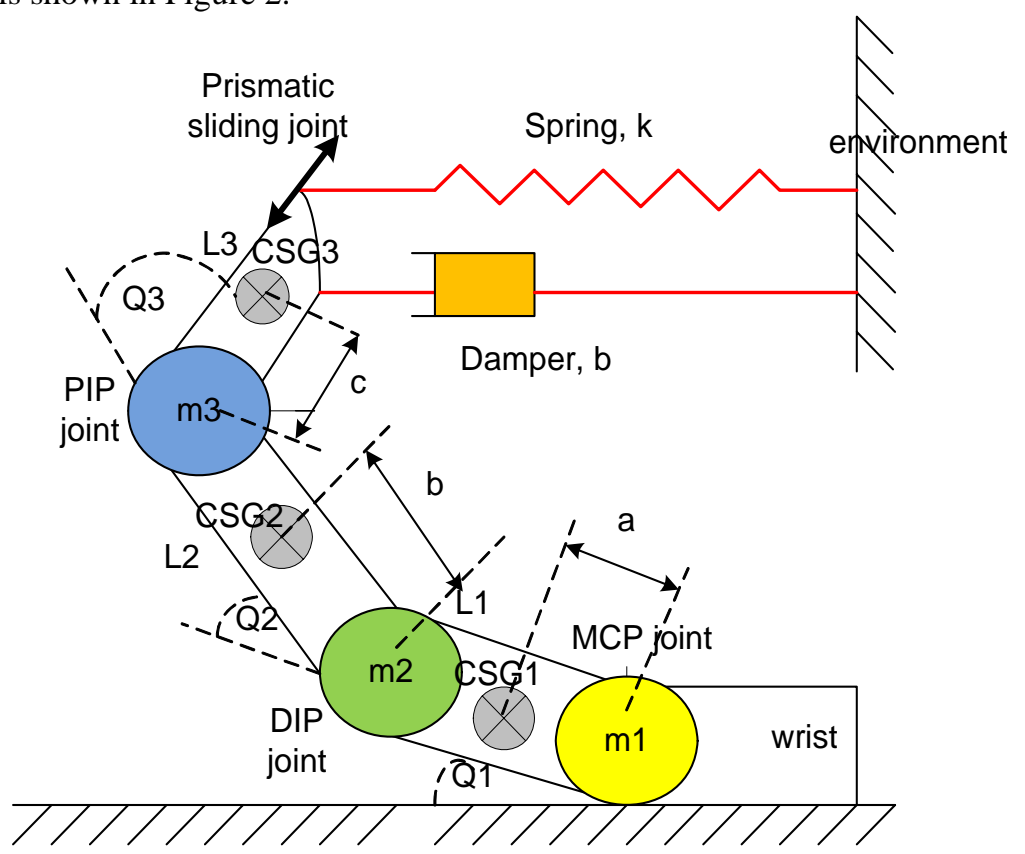

Figure 2 Finger model of robot hand. 
The mass of body $m_{i}$, the length of link $L_{i}$ and the inertia mass moment of the related links $I_{i}$ are the set of parameters of a finger. a, b and c are the distances of the mass centre of the first, second and third link, respectively. $m_{l}$ represents the mass of the joints and $\vartheta_{i}$ is the joint angle of the related link.

The fingers dynamic equation can be expressed as,

$$
\tau(t)=H\left(\theta(t), \stackrel{0}{\theta}(t)+h(\theta(t), \stackrel{0}{\theta}(t))+G(\theta(t))+\tau_{\theta}\right.
$$

Where,

$\tau(t)$ is actuator torque vector in angle $\mathrm{i}$,

$H(\theta)$ is dynamics transform matrix vector (nxn) in n DOF,

$\theta$ is the n vector of joint displacements, $\mathrm{n}$ being the number of finger axes,

$0 \quad 00$

$\theta, \theta$ are respectively the vectors of joint velocities and accelerations,

$h(\theta)$ is torque matrix vector (nx1) of Coriolis force effect and centrifugal motion in angle i,

$H(\theta)$ is gravitation effect torque matrix vector (nx1),

$\tau_{\theta}$ is disturbance torque vector.

A mass spring damper is acted at end of finger tips to connect with environment. By Newton's third law, the spring applies equal and opposite force to the two bodies. In the translational joint, the displacement $\mathrm{x}$ along a prismatic axis, and $v \mathbf{m} \alpha x \xi \delta$ is the prismatic DoF's linear speed, then the damped spring force acting along this prismatic and between the bodies connected by joint is

$$
F=-k\left(x-x_{0}\right)-b v
$$

The model parameters are the spring $\mathrm{k}$, the natural spring length (offset) $X_{0}$, and the damping constant $\mathrm{b}$.

2.4 Actuation Mechanism: A DC-Servo motor (DC-SV) is assumed as a pure torque motor. The advantage of a servo motor is precise rotational control which is based upon the number of steps/rev and its associated gearing. Rotational control is undertaken by a rotational potentiometer connected to the shaft. The feedback is used as a signal to a motor controller which will stop the rotation of the shaft via control of the particular motor in use.

\section{Physical Modelling}

Using SimMechanics, it becomes possible to model and simulate mechanical systems with a suite of tools to specify bodies and their mass properties, their possible motions, kinematic constraints and coordinates system and to initiate and measure body motions (Wood et al, 2003). The three fingers gripper assembly was built in Solidwork tool, and it's mechanical assembly representation exported using SimMechanics. The CAD assembly in Solidworks program is automatically changed to a physical model in XML for import to SimMechanics software (Mathworks, 2009). The physical model was created using SimMechanics which assumes that all systems are made of bodies with different DOFs, specific positions, orientations and masses. This model can be used to simulate flexion motion of the fingers. The wrist is modelled by a solid which provides no DOF, the MCP joint is modelled using a revolute block which provides only one DOF (no adduction /abduction motion). The PIP and DIP joints are also modelled using revolute blocks. The palm and the phalanges are modelled using body blocks, which provide the orientation with respect to the general coordinate system (the ground block), the length, the mass, etc. The physical SimMechanics model of one joint of finger is shown in Figure 3. 


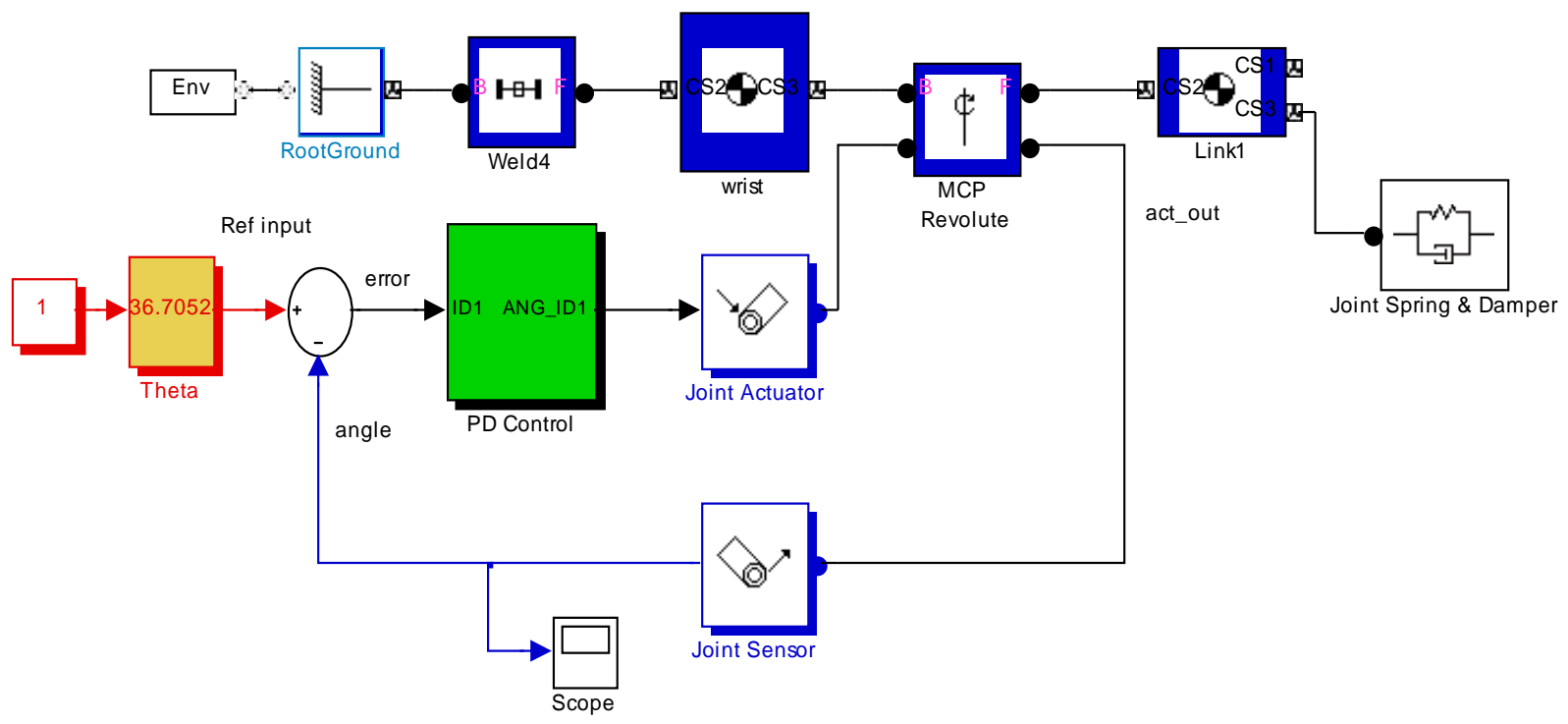

Figure 3 The abstraction of Physical modelling of MCP thumb gripper

In this model has machine environment block (Env), ground block, two bodies block as wrist and arm, revolute joint and weld joint, joint actuator, sensor block and joint spring and damper. The machine environment allows us to view and change the mechanical environment setting for one machine in our model. The Env block is connected to the ground block, which allows us to input gravity as a variable simulink signal. Weld block represents a joint with no degrees of freedom. Two bodies connected to either side of the Weld block are locked rigidly to one another, with no possible relative motion. The body block represents a rigid body with properties. Each body of the model has mass and moment of inertia tensor, the coordinates for the body's centre of gravity (CG) and its own co-ordinate system (CS), which is expressed with respect to the CS of the body immediately placed before it. Every joint has to be actuated using a joint actuator block, which provides the value of the rotation angle. The motion of the fingertip is captured using a position sensor block and plotted using a scope block. Using SimMechanics can be applied to define forces in joint friction and the joint spring. In addition SimMechanics also features a tunable contact model for which the contact spring rate and damping for the joint limits can be attached. The spring mass damper block is installed in the prismatic joint to compute the applied force when one finger is contacted with others. Suitable values of spring and damping constants are set up in the spring mass damper block.

\section{Control of Fingers}

Position control is used when the robot finger must be moved with or without load along a prescribed trajectory through the robot finger workspace. The control system of robot fingers is simply a collection of joint controllers each of which is dedicated to a single joint and drives it individually. The reference signals for these controllers are supplied by a joint trajectory generator determining the desired joint trajectory from the desired trajectory of the gripper. The joint controller is a feedback controller having two terms respectively proportional to position and velocity errors and introducing proportional and derivative actions (P-D control). 


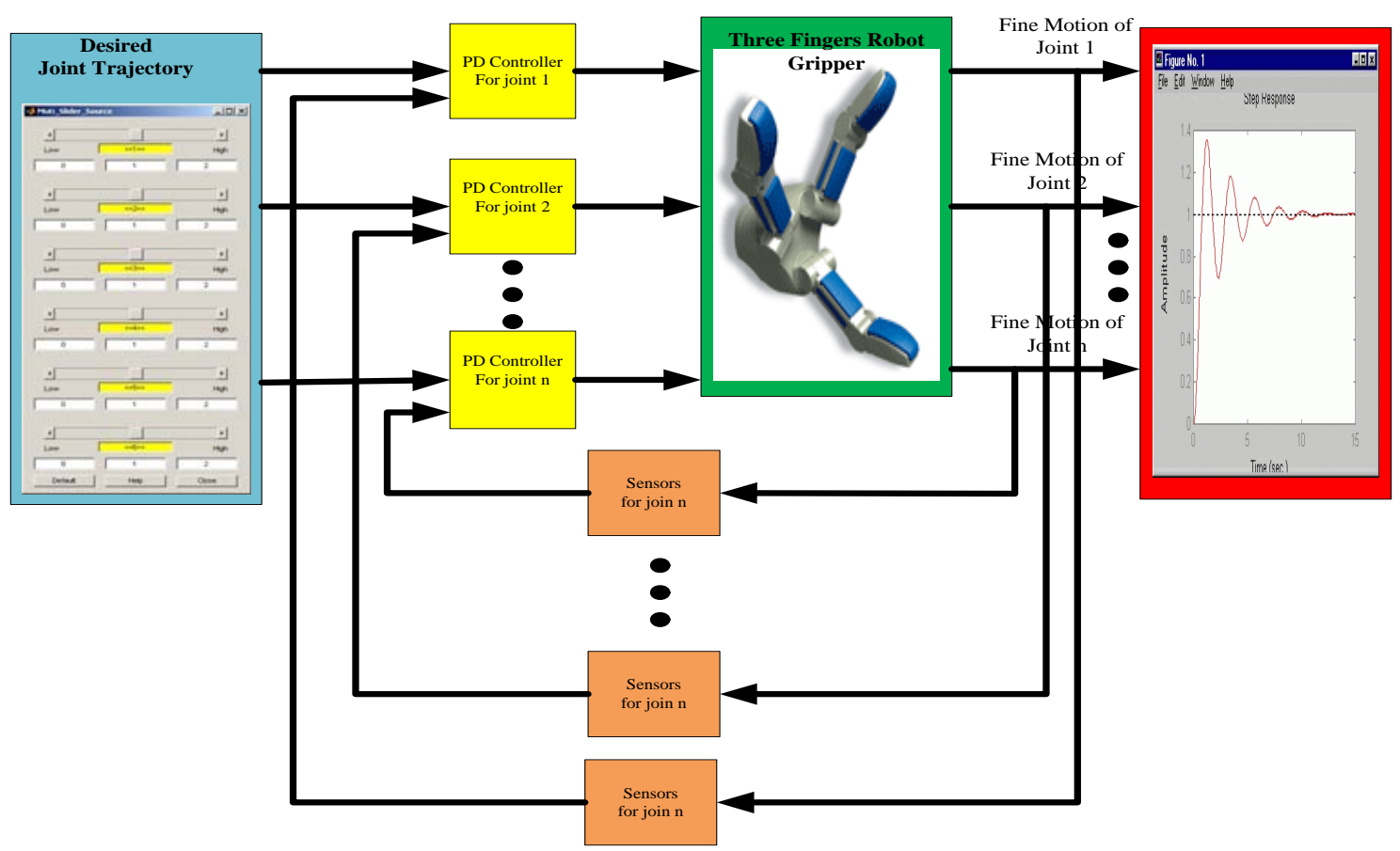

Figure 4 Control system of a n-DOF Fingers Robot Gripper

Standard PD control has been applied to control the motion positions, angular velocities and angular accelerations in the joint angles of each of the fingers and applied force motion. By tuning suitable values of the two constant gains in the PD controller algorithm, the controller has been found to provide the necessary control action for specific process requirements. The suitable gains of Proportional gain (Kp) and Differential gain $(\mathrm{Kd})$ value are determined by manual tuning to achieve a fast response to steady state (setting tune) without excessive overshoot. The error signal is the difference between reference input angle $\left(\stackrel{o}{\theta_{r e f}}\right)$ and actual output angle $\stackrel{o}{\left(\theta_{\text {act }}\right.}$ ) of the system. Parameter Kd works on the change of the error signal, so it can reduce dramatically overshoot effects to reach steady state response. The PD motor control is illustrated in the diagram in figure 5. The output controller equation of $\mathrm{u}(\mathrm{t})$ as,

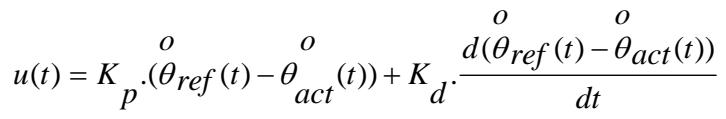

Based our previous research on control and simulation of three fingers gripper using a classical PID control, the transient response of motion was considered too slow (Widhiada et al, 2010). Choosing an incorrect value of integral gain caused an unstable signal response in the system with high oscillation. The motor and finger dynamics include some integral effects, hence it was possible to achieve low or zero steady state error without integral control action.

The command of the robot gripper is expressed in Cartesian coordinates of the finger gripper. Using the inverse kinematic problem formulation, these coordinates become displacements which are used as the reference points for the control algorithm. A proportional and derivative controller is designed for positioning control of the robotic finger.

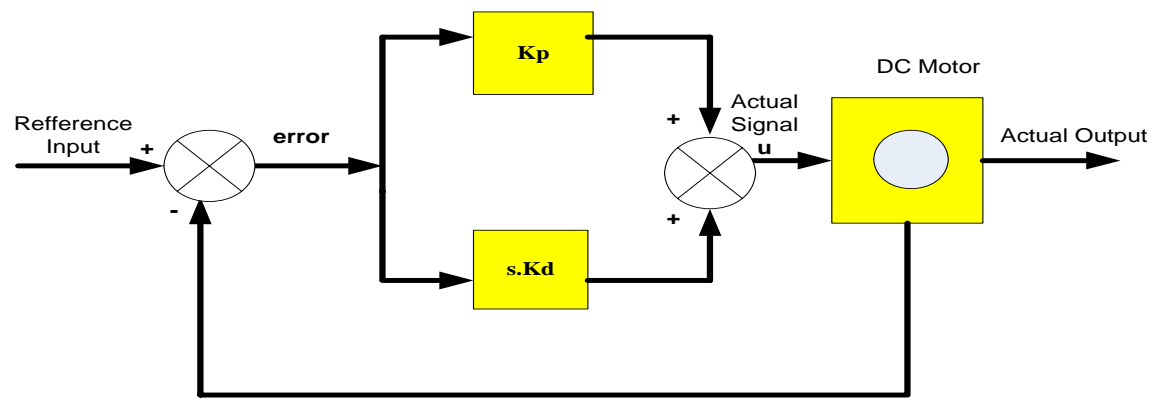

Figure 5 PD Control Diagram 
Two suitable constant gain values for PD control of the robot finger used in the simulation are given in Table 1.

Table 1. Gains used in simulation.

\begin{tabular}{|l|l|l|}
\hline \multicolumn{1}{|c|}{ Constant Gain } & \multicolumn{1}{|c|}{$\mathrm{Kp}$} & \multicolumn{1}{c|}{$\mathrm{Kd}$} \\
\hline Thumb & 50 & 70 \\
\hline Middle Finger & 50 & 70 \\
\hline Index Finger & 50 & 70 \\
\hline
\end{tabular}

The prismatic sliding element is attached at each fingertip where it can translate up and down. The extension element is used to pick up and manipulate small awkward elements such as rubber seals and springs typically found in a domestic gas regulator. We can control the displacement $(\mathrm{x}$ ) of the sliding extension using PD control. The actual desired response of the prismatic sliding element is shown in the simulation. The PD control scheme is linked to the joint actuators.

In this task there are three position configuration set points of finger which can operate by simulation in Matlab/SimMechanics. The reference of finger positions is show in Table 2 as below,

Table 2 Various gross motion for three fingers robot gripper

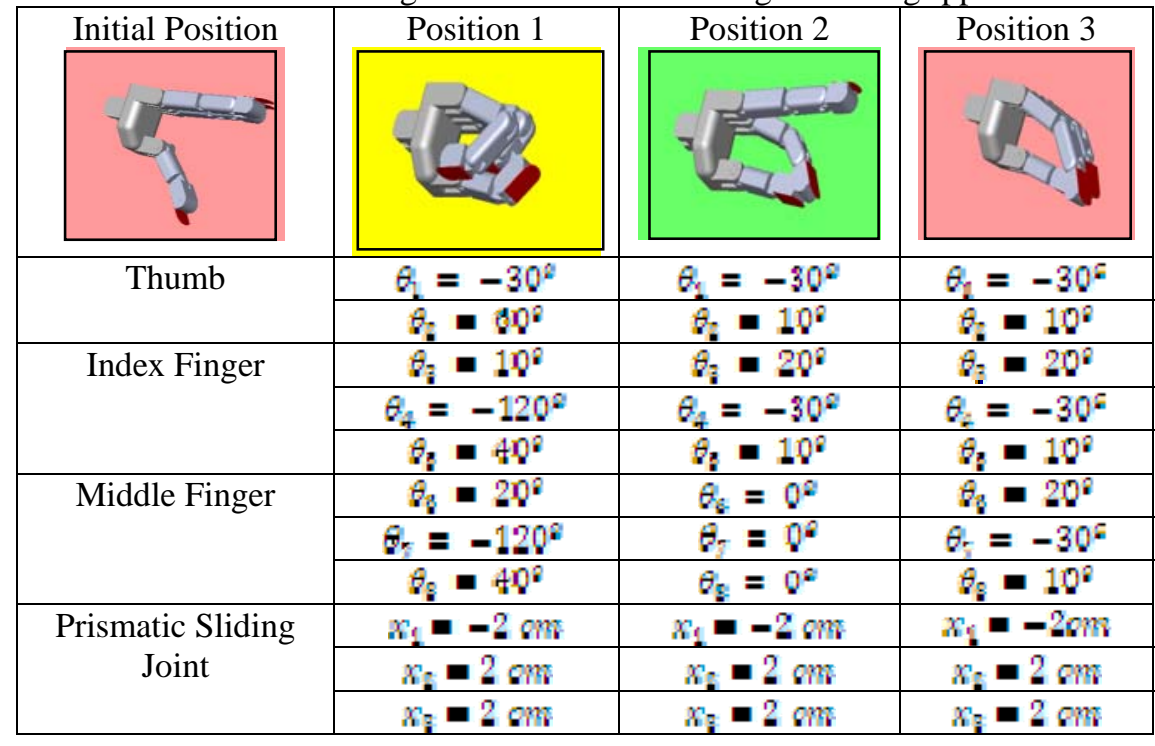

Where,

$\theta_{1,2,4, \pi, 5,8}$ are joint angle of three fingers Gripper in degree.

$x_{\text {: }: \text { a }}$ are the position of prismatic sliding elements in centimeter.

\section{Simulation Result}

A satisfactory of transient response for the finger simulation is achieved when the rise time is 0.0288 sec., setting time is $0.0784 \mathrm{sec}$., overshoot $10.3 \%$. The fine motions simulation results are presented in Figure 6 to 17 and are respectively for sensors in the MCP joints, PIP joints, DIP joints and the prismatic sliding joints. In addition the force simulation results are presented in Figure 18. In Figures 6, 7, 8 and 9 are simulation results for the first position of set point fingers. In Figure 6 show the desired stable angle for joint 1 and 2 of thumb are achieved smoothly in less than 0.1 sec and also in 0.1 sec the speed and angular acceleration was stable. In Figure 7 and 8 presented the desired fine motions in index and middle finger first position such angle, angular velocity and angular acceleration were stable in less than $0.1 \mathrm{sec}$. The first position for prismatic sliding element response is shown in Figure 9 and the desired translation of 0.02 is achieved smoothly in less than 0.1 sec. 

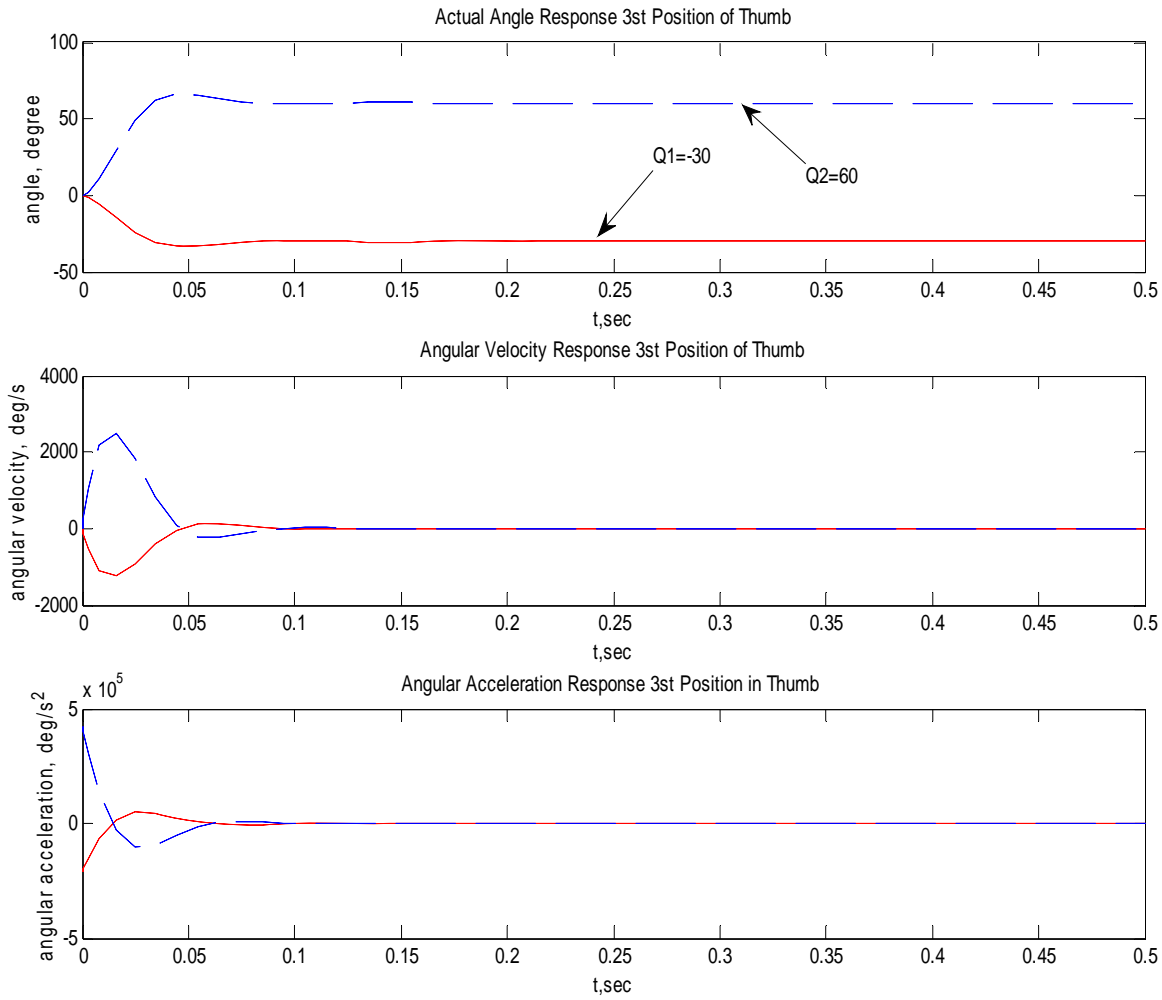

Figure 6 the $1^{\text {rt }}$ Position of Thumb Motions
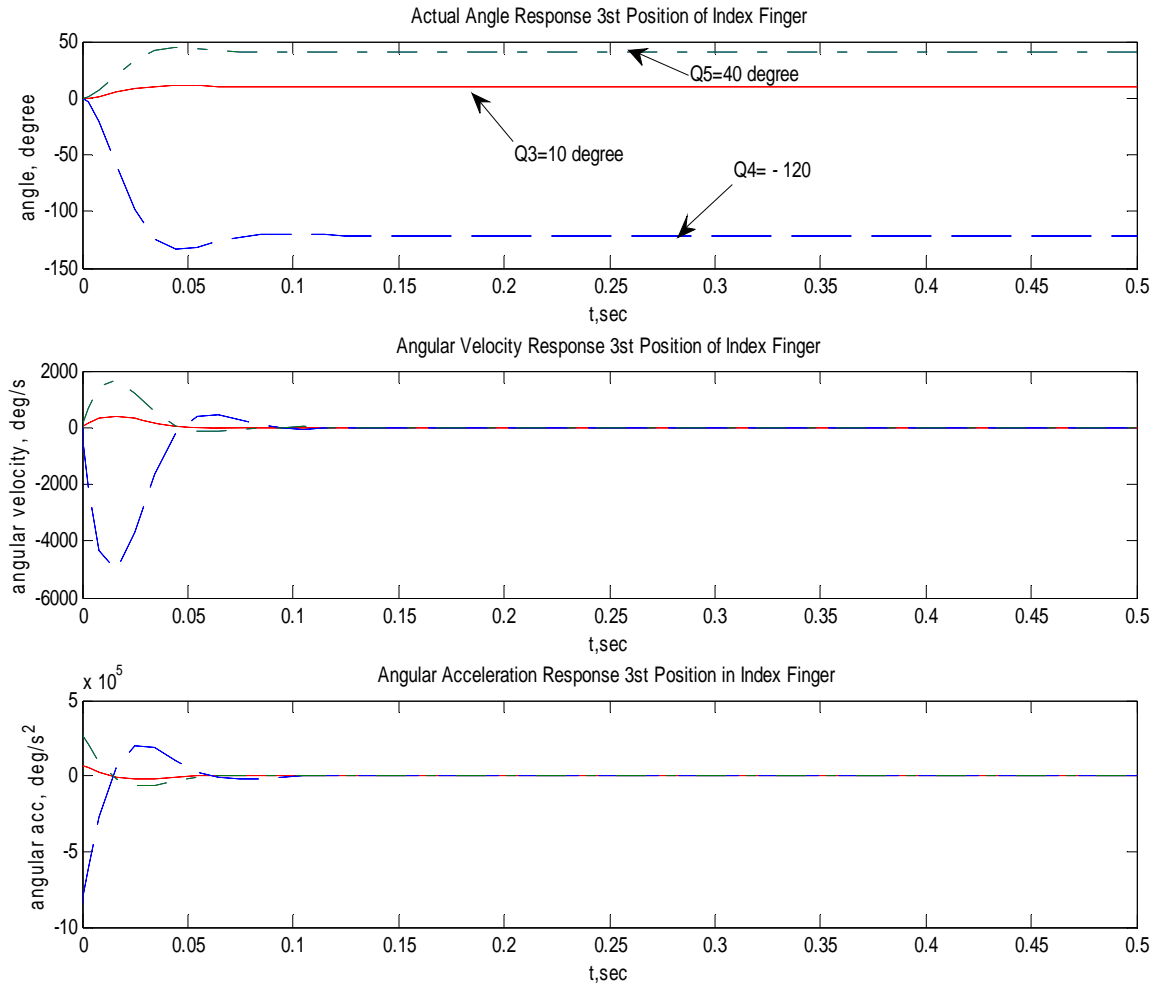

Figure 7 the $1^{\text {rt }}$ Position of Index Finger Motions 

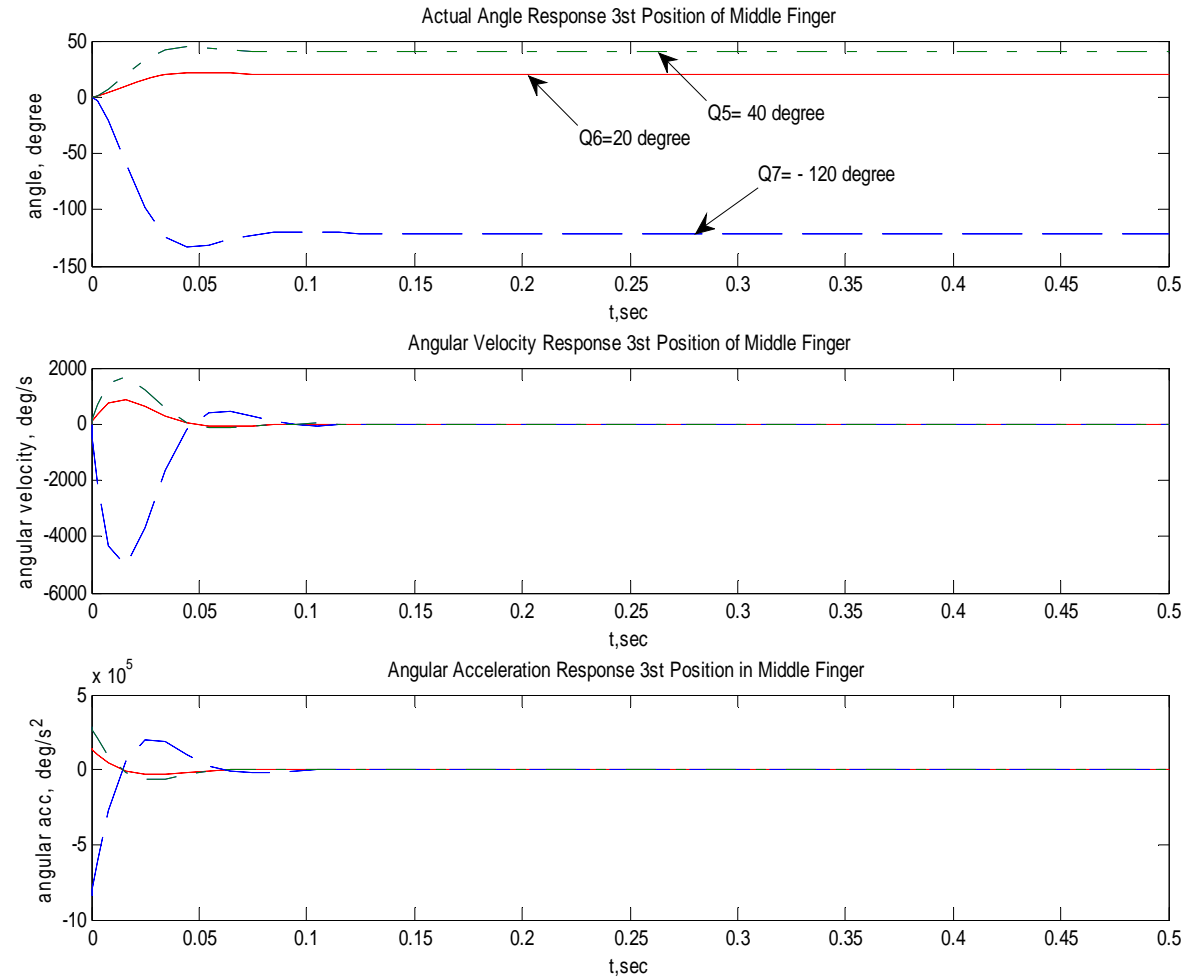

Figure 8 the $1^{\text {rt }}$ Position of Middle Finger Motions
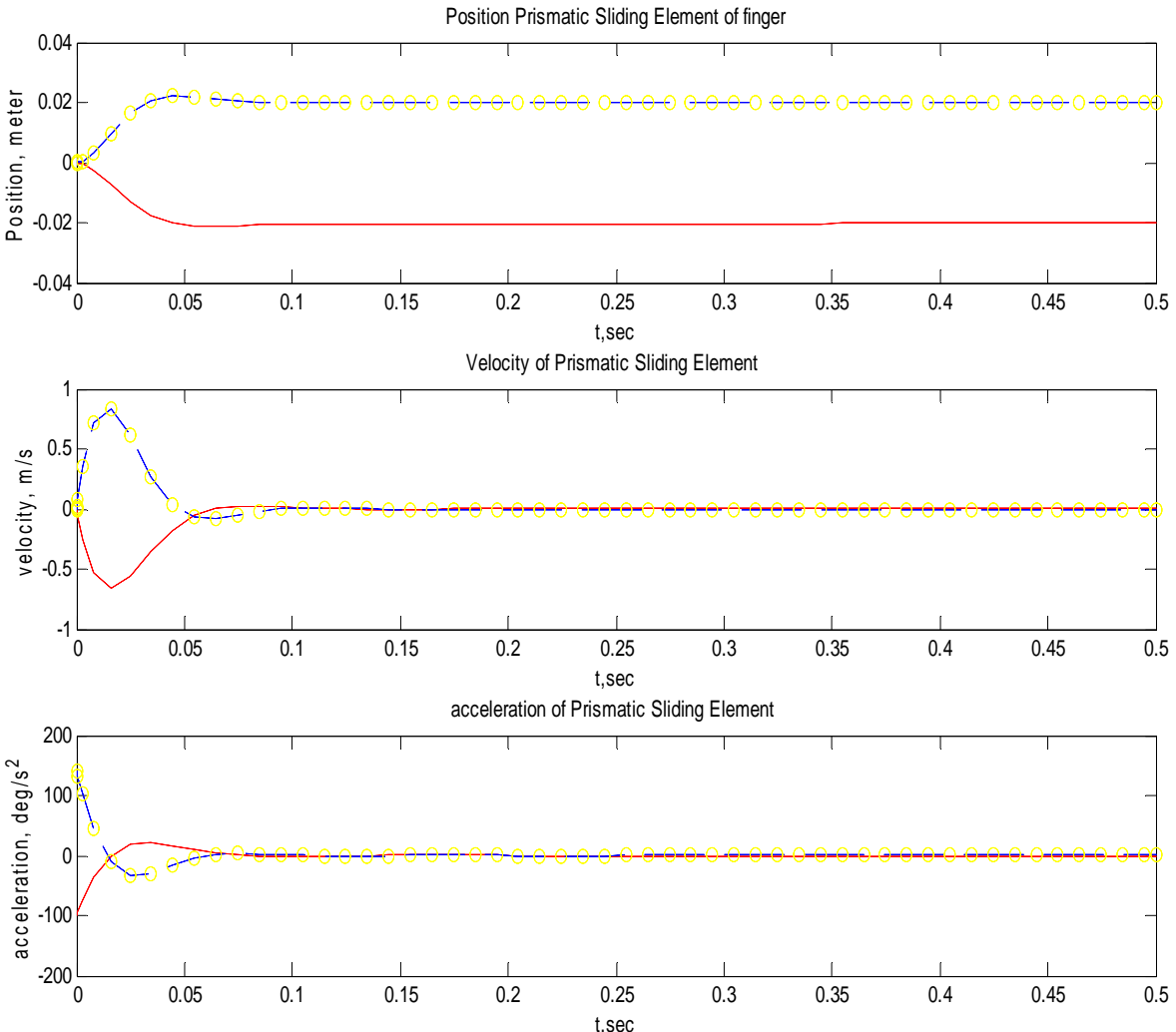

Figure 9 the $1^{\text {rt }}$ Position of Prismatic Joint Motions 
Figure 10, 11, 12 and 13 are simulation results for the second position of set point robot fingers. In Figure 10 show the desired angle for joint 1 and 2 of thumb are achieved smoothly in less than $0.1 \mathrm{sec}$ and also in $0.1 \mathrm{sec}$ the speed and angular acceleration was stable. In Figures 11 and 12 presented the desired fine motions for index and middle finger of first position in joint 3, 4, 5, 6, 7 and 8 and angular velocity and angular acceleration respectively were stable in less than 0.1 sec. The prismatic sliding element response is shown in Figure 13 and the desired translation of 0.02 is achieved smoothly in less than 0.1 sec.
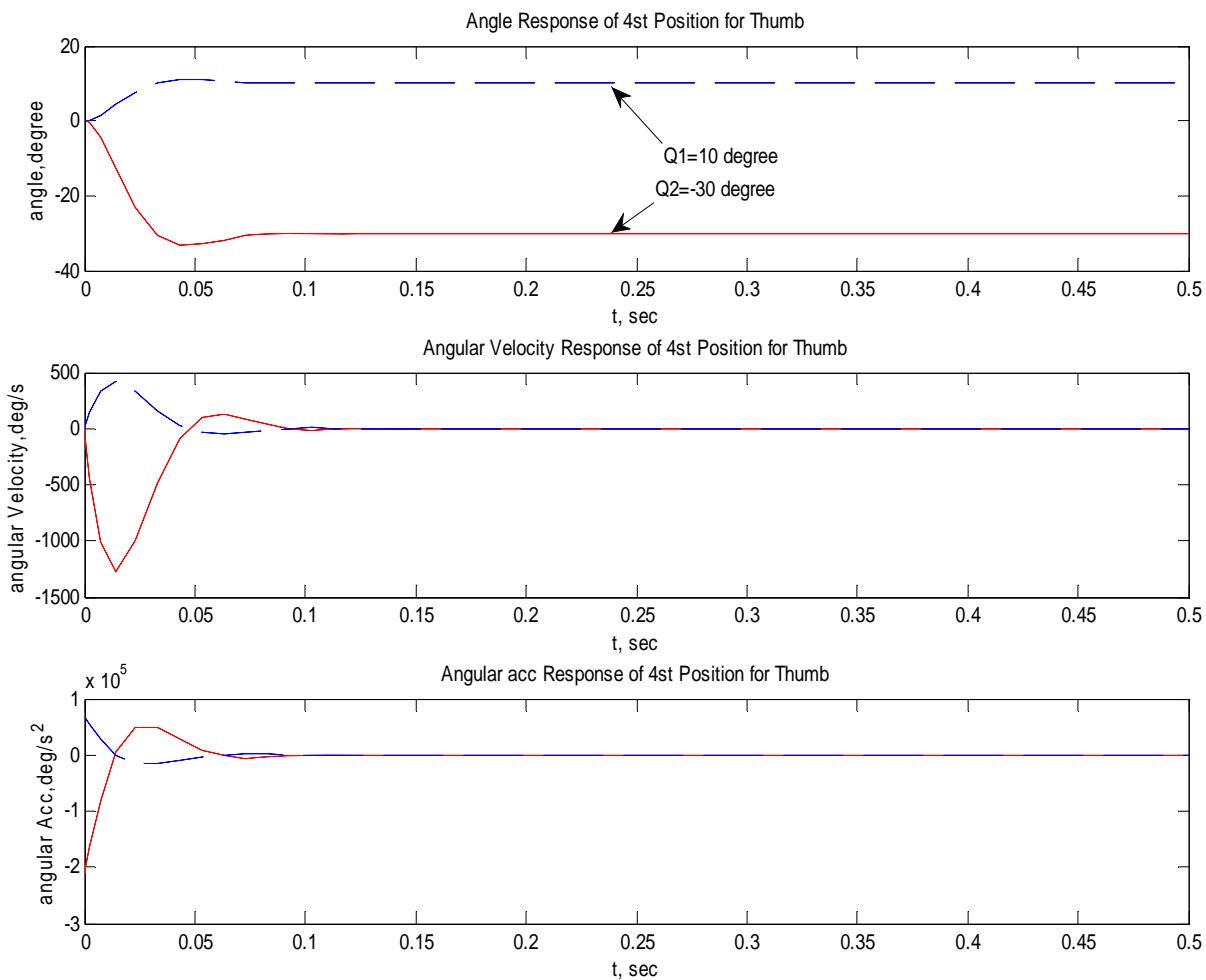

Figure 10 the $2^{\text {sd }}$ Position of Thumb motions. 

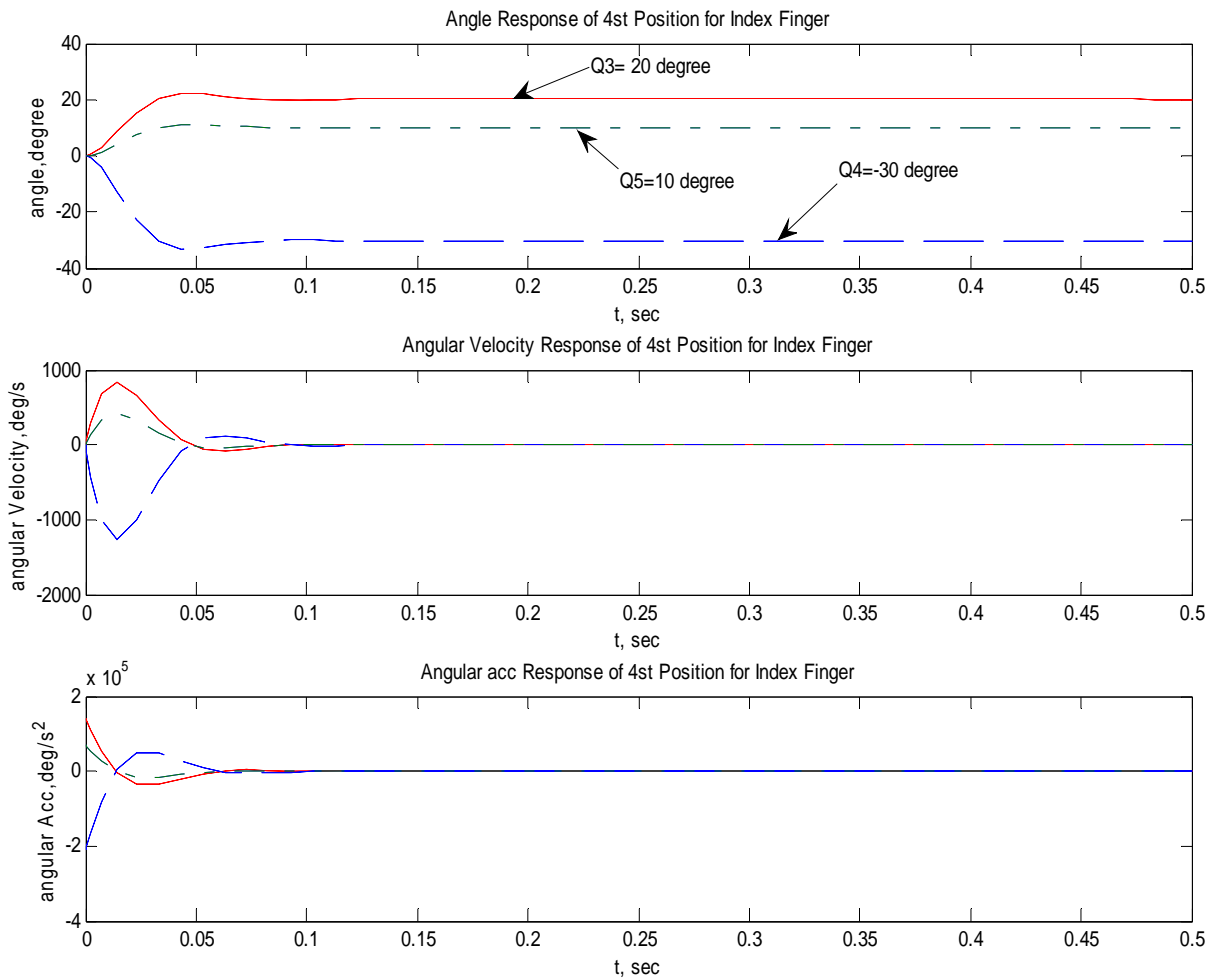

Figure 11 the $2^{\text {sd }}$ Position of Index Finger Motions


Figure 12 the $2^{\text {sd }}$ Position of Middle Finger Motions 

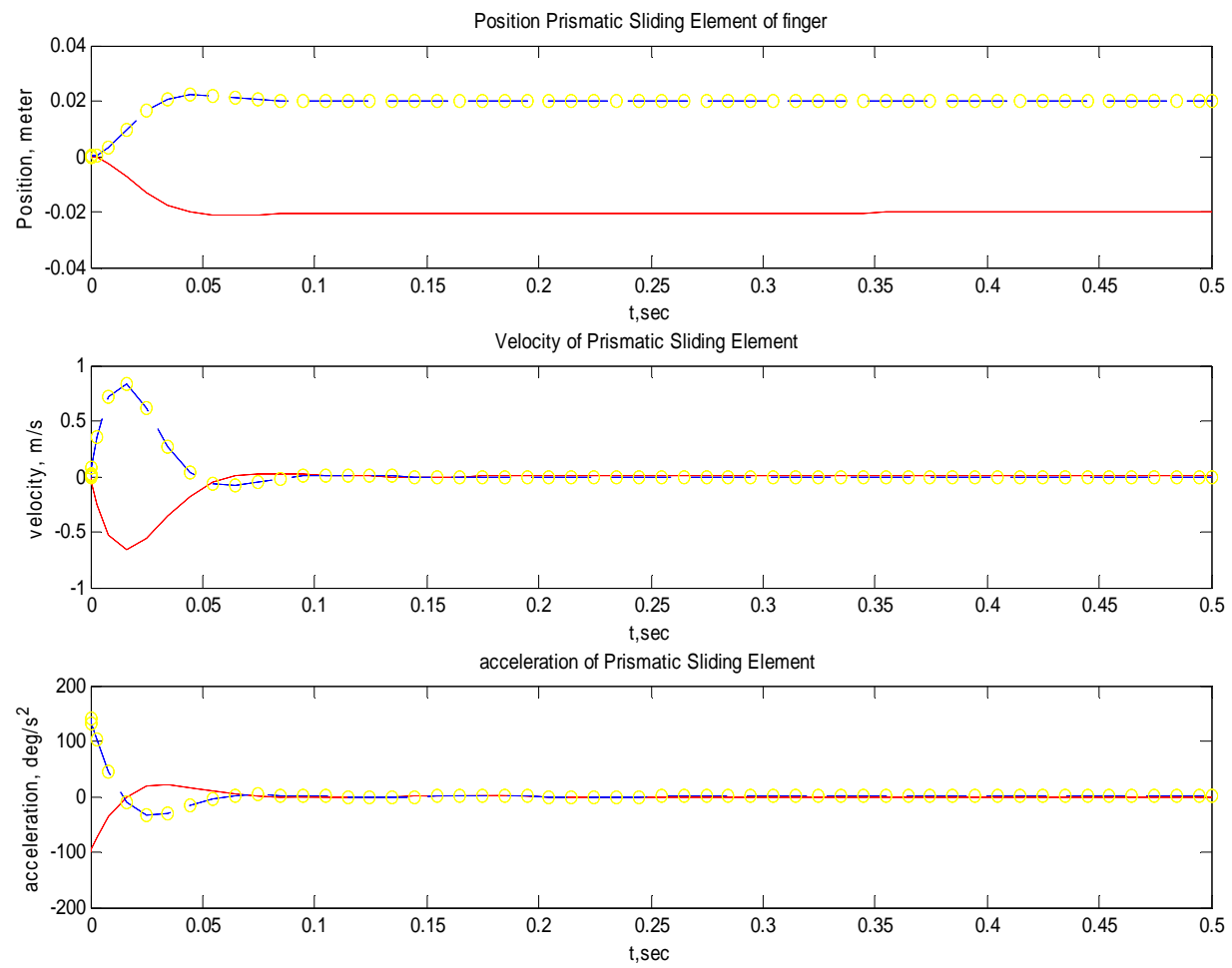

Figure 13 the $2^{\text {sd }}$ Position of Prismatic Joint Motions

Figure 14, 15, 16 and 17 are simulation results for the third position of set point configuration robot fingers. In Figure 14 shows the desired stable angle for joint 1 and 2 of thumb are achieved smoothly in less than $0.1 \mathrm{sec}$ and also in $0.1 \mathrm{sec}$ the speed and angular acceleration was stable. In Figures 15 and 16 presented the desired fine motions in index and middle finger first position such angle, angular velocity and angular acceleration were stable in less than $0.1 \mathrm{sec}$. The first position for prismatic sliding element response is shown in Figure 17 and the desired translation of 0.02 is achieved smoothly in less than 0.1 sec.
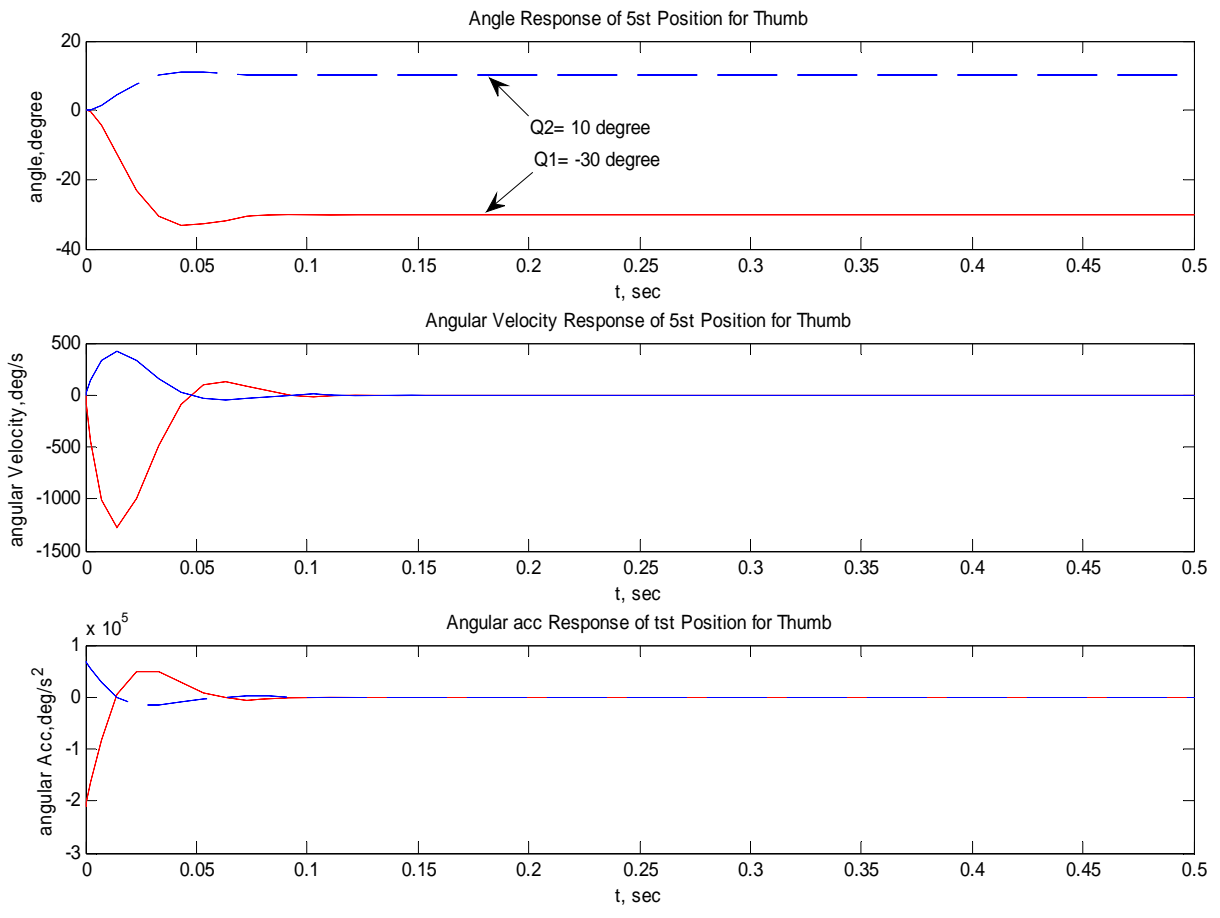

Figure 14 the $3^{\text {rd }}$ Position of Thumb Motions 



Figure 15 the $3^{\text {rd }}$ Position of Index Finger Motions
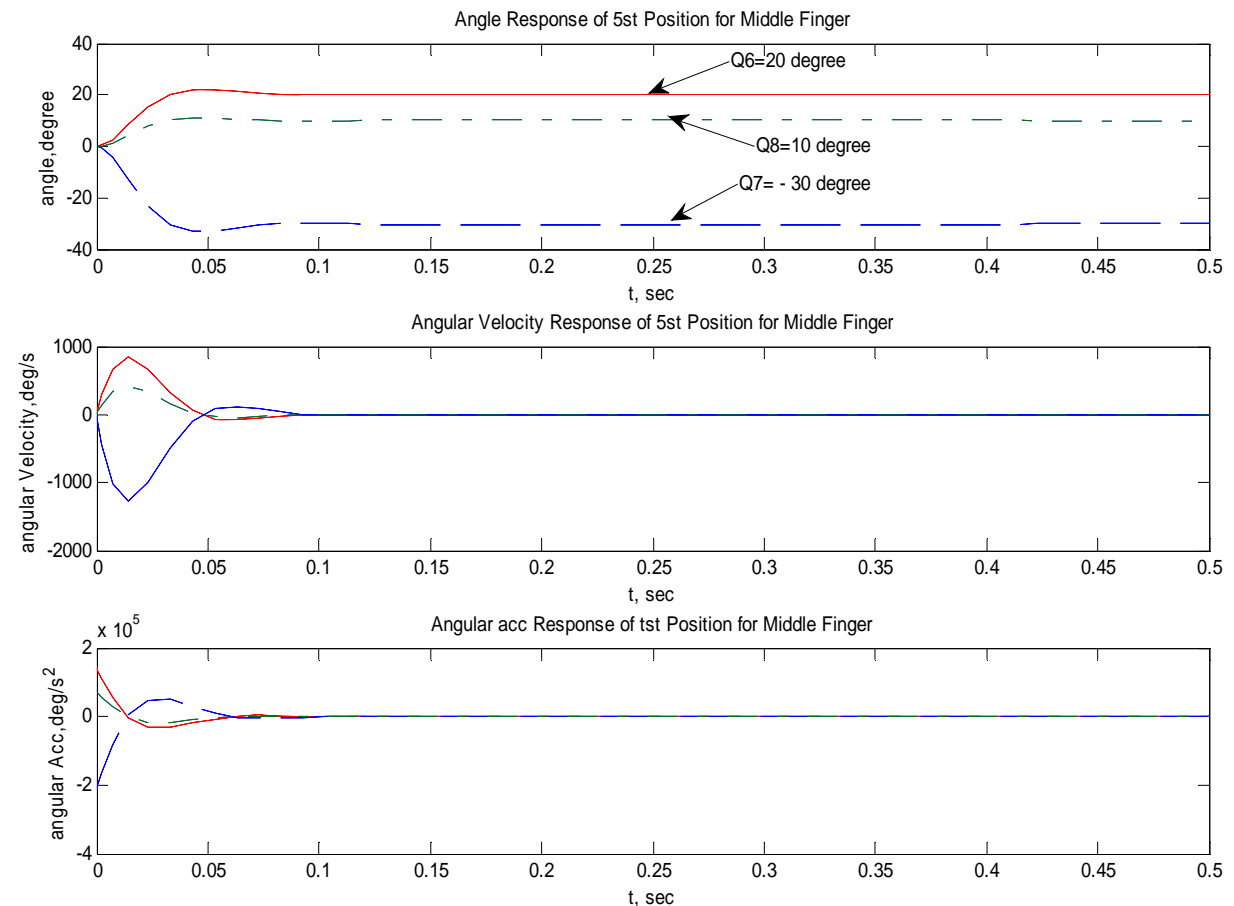

Figure 16 the $3^{\text {rd }}$ Position of Middle Finger Motions 

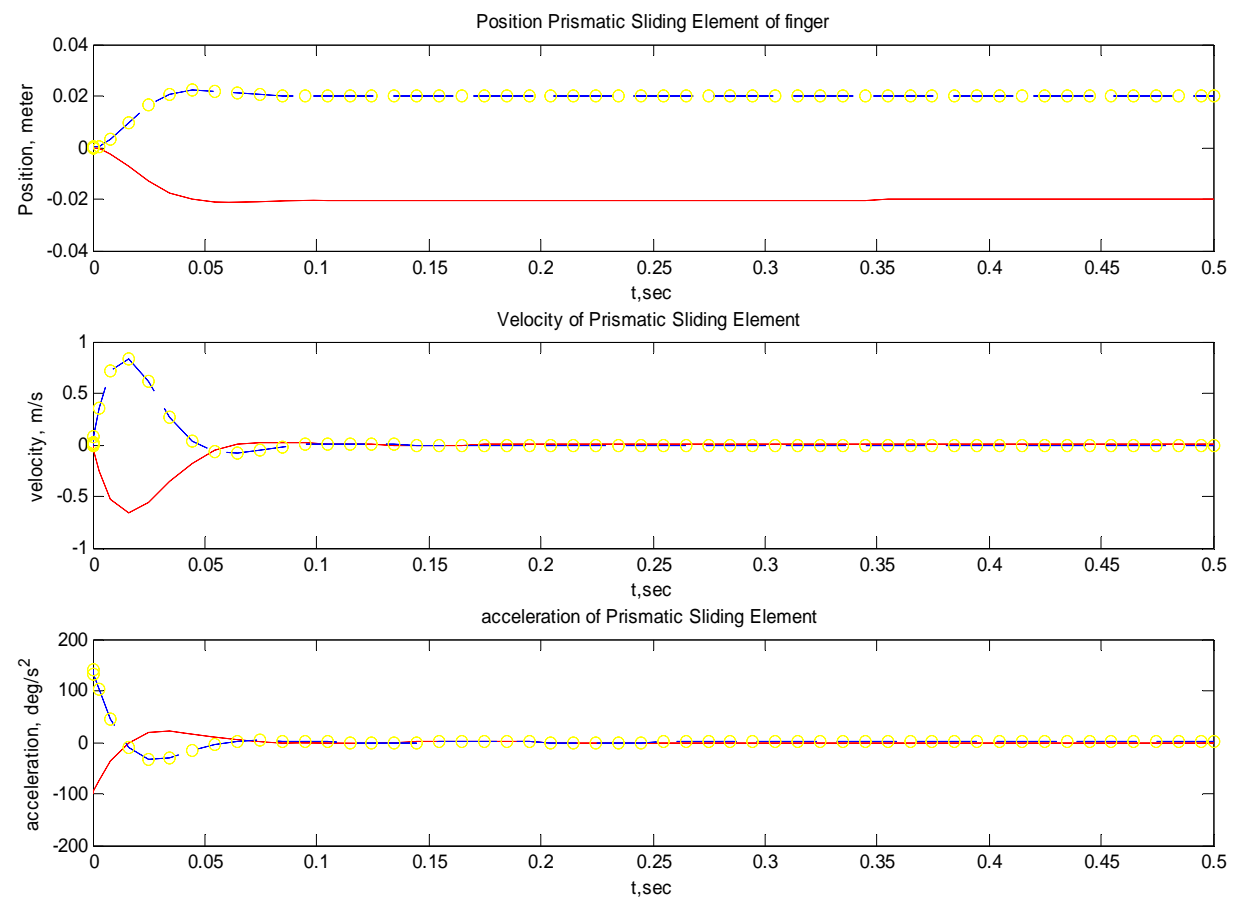

Figure 17 the $3^{\text {rd }}$ Position of Prismatic Joint Motions

Figure 18 presented the simulation and control of force at the end each finger. The calculation of the amount of force applied at the tip end of the thumb and index finger are the same at $21.6619 \mathrm{~N}$ respectively in the first position, $18.5 \mathrm{~N}$ in the second position and $18.5 \mathrm{~N}$ in the third position and achieved smoothly lest than in $0.138 \mathrm{sec}$. The amount of force applied at the tip end of the middle finger is at $21.9639 \mathrm{~N}$ respectively in the first position, $19.9577 \mathrm{~N}$ in second position and $18.05 \mathrm{~N}$ in the third position. 


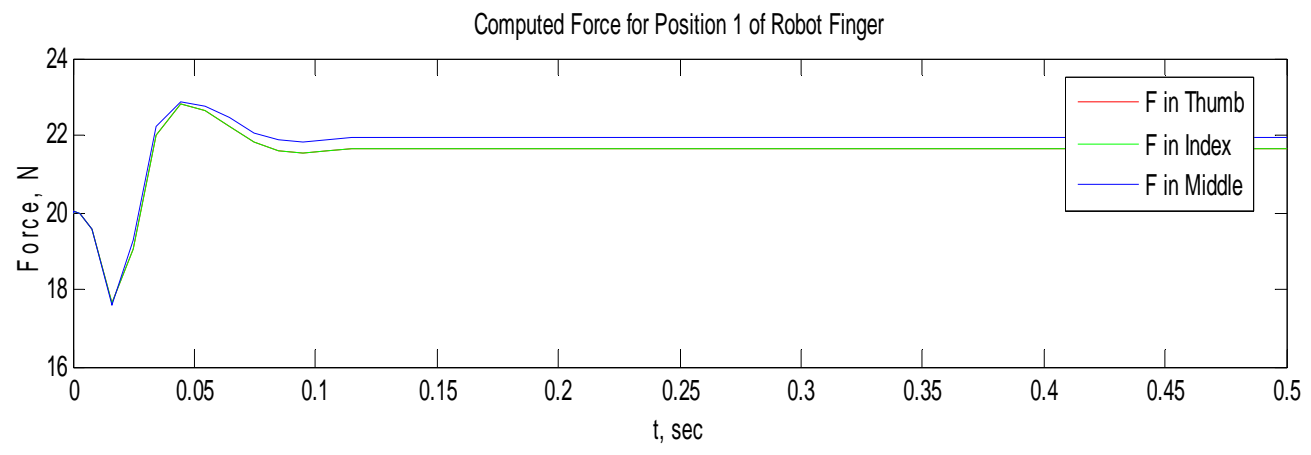

Computed Force for Position 2 of Robot Finger

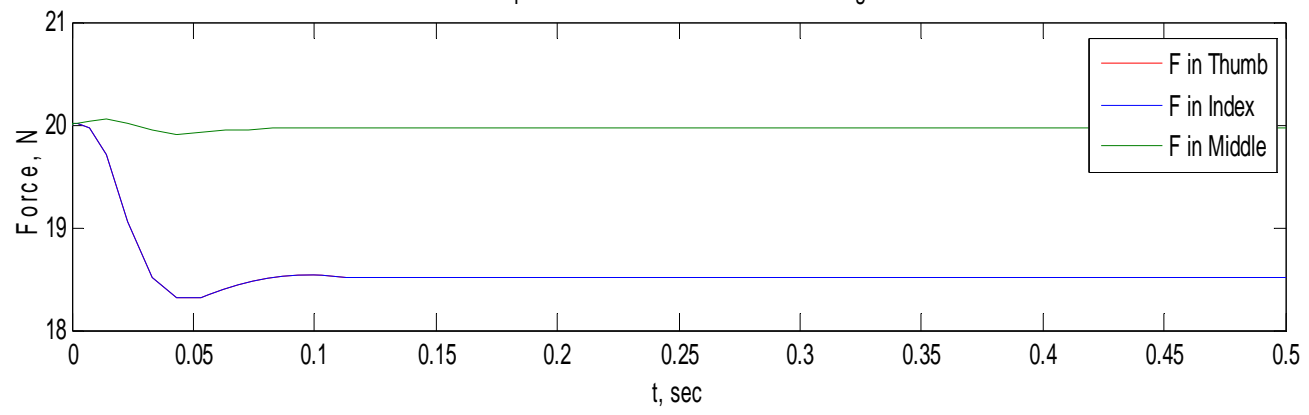

Computed Force for Position 3 of Robot Finger

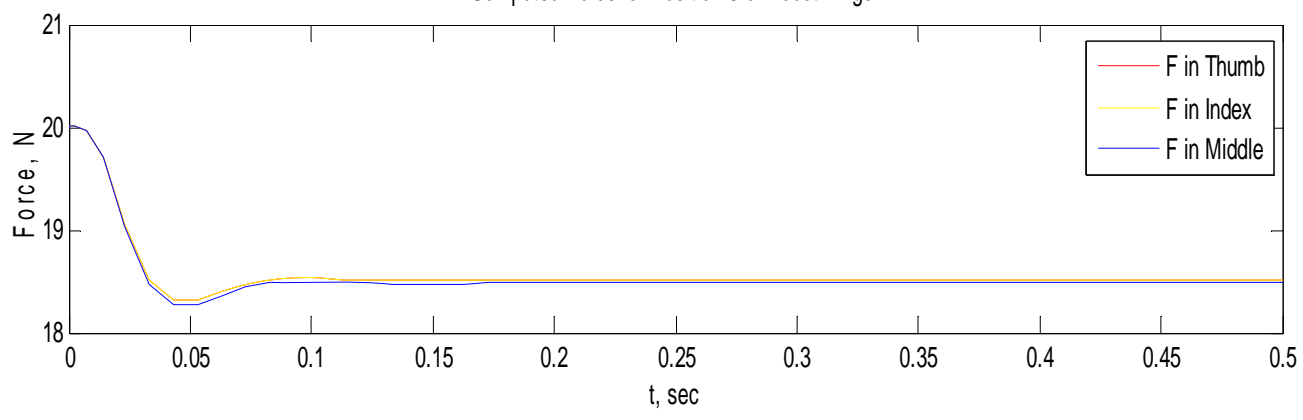

Figure 18 Simulation and control Force in the Position 1, 2, and 3 of Finger

\section{Conclusion}

The physical model of the three finger robot gripper is built using SimMechanics software and the application SimMechanics has also been verified with identical outputs when compared with Simulink simulations. These studies indicate that each finger of the gripper can be controlled using a PD formulation. A novel feature of this gripper is the inclusion of a prismatic sliding element at the end of each finger to facilitate the grippers handling of both large and small compliant components typically found in high speed the dexterous assembly of a domestic gas regulator. PD control is very effectively used to control the trajectory of the fingers and force used to hold objects. The simulation results have been demonstrated that the radius of finger movement is achieved in a very fast and smoothly stable. The fingers must be controlled in a way that mimics the kinematics and dynamics of force thumb and index finger of human hand. Mimicry is necessary to design the right motions and tactile forces necessary to handle the delicate and non delicate engineering components. In the future works, the dexterous grasping of compliant elements will be simulated to evaluate the stability of contact force between fingers and object environment. Cam law design methods will be investigated to obtain the dexterity of grasping objects.

\section{Acknowledgment}

Authors are grateful to the Higher Education Directorate General, Ministry of National Education, Republic of Indonesia for the financial support of this project. 


\section{References}

Akin,D.L.,Carigman,C.R., and Foster,A.W. Development of a four fingered dexterous robot end-effector for space operations. Proc. IEEE Int. Conf. Robotics and Automation, Washington, DC, 2002, Vol.3, pp. 2302-2308.

Bekey,G.,Tomovic,R. and Zelkjkovic,I. Control Architecture for the Belgrade/USC Hand in Dextrous Robot Hands, New York: Springer-Verlag, 1999, pp.136-149.

Bicchi,A. and Kumar,V. Robotic grasping and contact. Proc. IEEE Int. Conf. Robotics and Automation,2000, Vol. 1, pp.348

Biagotti,L.,Melchiorri,C., and Vassura,G. Control of a robotic gripper for grasping objects in no-gravity conditions. Proc. IEEE Int. Conf. Robotics and Automation, Seoul, Korea, 2001, Vol.2, pp. 1427-1432.

Crisman,J., Kanojia,C. and Zeid,I. A flexible, easily controllable robotic hand. IEEE Robot. Automat. Mag., 1996, Vol.3, pp. 3238.

Cutkosky M.R., On grasp choice, grasp models, and the design of hands for manufacturing tasks, IEEE Trasactions on Robotics and automation, Vol.15, No.3, June 1989.

Cole A.B.A., Hauser J.E, and Sastry S.S. Kinematics and Control of Multifingered Hands with Rolling Contact. IEEE Trans on Automatic Control, 1989, Vol. 34, No. 4, pp. 398-404.

Dechev,N.,Cleghom,W. and Nauman,S. Multiple finger, passive adaptive grasp prosthetic hand, Mech. Mach. Theory, 2001, Vol. 36, pp.1157-1173.

Gazeau,J. Force and position control in the aim of the fine manipulation of objects. Proc. IEEE Int. Conf. Robotics and Automation, Seoul, Korea, 2001, Vol.3, pp. 2642-2648.

Guerry,M. Proper gripper selection is key to trouble-free operation. Robotics World, 2001, Vol. 19, No. 1, pp. 32-35.

Hirose,S. and Umetani,Y. The development of soft gripper for the versatile robot hand, Mech. Mach. Theory, 1978, Vol. 13, pp. 351-358.

Hirose,S. And Umetani,Y. The development of soft gripper for the versatile robot hand, Proceeding of the seventh International Symposium on Industrial Robots,1977, pp.353-360.

Jacobsen, S., et al. Design of the Utah/MIT dextrous hand. Proc. IEEE Int. Conf. Robotics and Automation, San Francisco, CA, 2001, vol.3,pp. 1520-1532.

Ji.Z., and Roth B., Direct Computation of Grasping Force for Three-Finger Tip-Prehension Grasps, ASME Trans. on Mechanisms, Transmissions, and Automation in Design, 1988, Vol. 110, pp. 405-413.

Johan,T, et al, Demontration-based learning and control for automatic grasping, Springer-verlag, 2008.

Kerr.J. and Roth B., Analysis of Multifingered Hands”, Int. Journal of Robotics Research, 1986, Vol. 4, No. 4, pp. 3-17.

Kobayashi H. Control and Geometrical Considerations for an Articulated Robot Hand, Int. Journal of Robotics Research, 1985, Vol. 4, No. 1, pp. 3-12.

Kolpanshnikov,S. And Chelpanov,I. Defining of the scheme and the parameters of an industrial robot gripper by a group of specifications, Proceeding of the $11^{\text {th }}$ International Symposium on Industrial Robots, Tokyo,Japan,1979, pp.301-308.

Kyriakopoles,J.K, Riper,J.V., Zink, A.et al. Kinematic analysis and position force control of the anthrobot dexterous hand, IEEE Transaction on System, Man and Cybernetics-Part B:Cybernetics, 1977, Vol.27 No.3,pp.257-263.

Lakshiminarayana.K. Mechanics of Form Closure, ASME Technical Paper, 1978, 78-DET-32.

Lee,J. Kinematic synthesis of industrial robot hand/gripper, Robotics, 1982, Vol.4 No.3, pp.257-263.

Leon, Z., Simulation in Robotics, Science Direct-Mathematics and Computer Simulation 79, pp. 879-897, 2008

Li.Z. and Sastry S.S. Taks-Oriented Optimal Grasping by Multifingered Robot Hands, IEEE Trans. Robotics and Automation, 188, 4(1):32-44.

Lunstrom, G. Industrial robot grippers, Industrial Robot, 1977, Vol.1, pp.72-82.

Massa,B. At al. Design and development of an underactuated prosthetic hand. Proc. IEEE Int. Conf. Robotics and Automation, Washington, DC, 2002, pp. 3374-3379.

Mathworks, SimMechanics Translators: http://www.mathworks.com/products/simmechanics/description5.html, 2009.

Nakamura Y., Nagai K., and Yoshikawa T. Dynamics and Stability in Coordination of Multiple Robotic Mechanisms, Int. Journal of Robotics Research, 1989, Vol. 8, No. 2, pp. 44-61.

Nguyen V. Constructing Force-Closure Graspes, In Proc. IEEE Int.Conf. on Robotics and Automation, 1986, pp. $1368-1373$.

Nguyen V. Constructing Force-Closure Graspes in 3D, In Proc. IEEE Int. Conf. on Robotics and Automation, 1987, pp. $240-245$.

Nguyen V., Constructing force-closure grasps, Int. Journal of RoboticsResearch, 1988, Vol. 7, No. 3, pp. 3-16.

Ohol S.S and Kajale,S.R. Simulation of Multifinger Robotic Gripper for Dynamic Analysis of Dexterous Grasping, Proceedings of the World Congress on Engineering and Computer Science, October 22-24 2008,. pp.908-914.

Okada,T. Computer control of multijointed finger system for precise object handling, IEEE Trans. Syst., Man, Cybern., Feb.1982, Vol. SMC-12, pp.289-299.

Okada,T. And Tsuchia,S. On versatile finger system, Proceedings of the Seventh International Symposium on Industrial Robots, 1977, pp.345-352.

Qiao,B.,et al, Learning Force for Position Controlled Robotic Manipulator, Nanjing University of Aeronautics and ustronautics Hina, 1999.

Reuleaux F. and.Kennedy A.B.W. Kinematics of Machinery, London: Macmillan and Co., access online, 1976.

Salisbury,J.K. and Craig,J. Force control and kinematic isues, Int..Root.Res.,1982, Vol.1,pp.4-17. 
Sastry S.S. and Z. Li. Robot Motion Planning with Nonholonomic Constraints, In Proc. IEEE Int. Conf. on Decision and Control, 1989, pp. 211- 216.

Schulz, S., et al. Design of UTAH/MIT dexterous hand. Proc. IEEE Int. Conf. Robotics and automation, San Fransisco, 2001, pp.1520-1532.

Seiichiro K, Matsumoto, Y. and Ohnisihi, K., Modeling of Force Sensing and Validation of disturbance observer for Force Control, IEEE Transactions on industrial electronics, vol.54 no.1, 2007.

Skinner,F. Designing a multiple prehension manipulator, Journal of Mechanical Engineering, 1975, Vol.97 No.9,pp.30-37.

Voshikawa T. and Nagai K. Manipulating and Grasping Forces in Manipulation by Multi-fingered Hands, In Proc. IEEE Int. Conf. On Robotics and Automation, 1987, pp. 1998-2004.

Widhiada, W, Douglas, S.S, Jenkinson, I and Gomm, J.B., Design of a novel robotic gripper for dexterous assembly of compliant elements, Proc. Of the $16^{\text {th }}$ International Conf. on automation and computing, Birmingham U.K., pp.297-302, 2010.

Wood G.D, and Kennedy D.C., Simulating mechanical systems in Simulink with SimMechanics, Technical report, the Mathworks, Inc, USA, 2003. http://www.mathworks.com

Yoshikawa T. and Nagai K., Evaluation and Determination of Grasping Forces for Multi-fingered Hands, In Proc. IEEE Int. Conf. on Robotics and Automation, 1988, pp. 245-248.

\section{Biographical notes}

W. Widhiada, S.S. Douglas and I.D.Jenkinson are of the School of Engineering Liverpool John Moores University, Byrom Street, Liverpool, L3 3AF, UK. In addition, W. Widhiada is also of the Department of Mechanical Engineering, Udayana University, Denpasar, Bali, INDONESIA.

Dr. J. Barry Gomm received the B. Eng. first class degree in electrical and electronic engineering in 1987 and the Ph. D. degree in process fault detection in 1991 from Liverpool John Moores University (LJMU), UK. He joined the academic staff at LJMU in 1991 and is a reader in intelligent control systems. His research interests include neural networks for modeling, control and fault diagnosis of non-linear processes, intelligent methods for control, system identification, adaptive systems, chemical process, and automotive applications.

Received December 2010

Accepted April 2011

Final acceptance in revised form July 2011 